\title{
Fourier Volume Registration based Dense 3D
}

\section{Mapping}

\author{
Luke Lincoln ${ }^{1, *}$, Ruben Gonzalez ${ }^{1}$ \\ ${ }^{1}$ Institute for Integrated and Intelligent Systems (IIS), Griffith University, Gold Coast, Australia
}

\section{Abstract}

In image processing phase correlation has been shown to outperform feature matching in several contexts. In this paper, a novel volume registration technique is proposed for solving the simultaneous localization and mapping (SLAM) problem. Unlike existing methods which rely on iterative feature matching, the proposed method utilises 3D phase correlation. This method provides high noise robustness, even in the presence of moving objects within the scene which are problematic for SLAM systems. Furthermore, a novel projection method is proposed which performs Fourier based volume registration 3 times faster. Quantitative and qualitative experimental results are presented, evaluating the proposed method's the noise sensitivity, performance, reconstruction quality and robustness in the context of moving objects.

Received on 16 May 2016; accepted on 27 June 2016; published on 12 September 2016

Keywords: SLAM, volume registration, phase correlation, Fourier transform

Copyright (C) 2016 Luke Lincoln and Ruben Gonzalez, licensed to EAI. This is an open access article distributed under the terms of the Creative Commons Attribution license (http://creativecommons.org/licenses/by/3.0/), which permits unlimited use, distribution and reproduction in any medium so long as the original work is properly cited.

doi:10.4108/eai.12-9-2016.151675

\section{Introduction}

Simultaneous localization and mapping (SLAM) has many applications in robotics, architecture and engineering, business and science. Its objective is to produce a map (2D birds-eye-view, or 3D) of an environment using image and other sensory data. This is typically performed by computing local features, iteratively matching them across frames and solving for the camera pose and location. This feature matching approach is dependent on finding a sufficient number of matches. When this is true the approach is able to cope with local matching disparities by treating them as outliers. This technique is not robust when features are not stable or when feature confusion occurs.

To alleviate these shortcomings we propose using $3 \mathrm{D}$ phase correlation based volume registration to solve the SLAM problem. Given two volumes, volume registration algorithms find a geometrical transformation in which to align the data within the volumes. Fourier based registration is known to be fast, robust to noisy data and scales naturally to

\footnotetext{
${ }^{*}$ Luke Lincoln. Email: luke.lincoln@griffithuni.edu.au
}

parallel processing [8]. It has also been shown to be able to outperform local feature based methods in 2D image processing [10]. This approach is capable of real-time SLAM if used in a parallel programming context. However, this approach is still computationally complex for most practical volume sizes on multiprogramming systems.

In the context of SLAM, scale is often not required in registering volumes. Therefore we propose a novel technique to speed up Fourier based volume registration for estimating translation and rotation parameters. This is achieved by applying a novel projection transform called a spherical-map transform. Along with orthogonal projection, this method allows $2 \mathrm{D}$ phase correlation to be used in place of the $3 \mathrm{D}$ counterpart. The result is a $3 \mathrm{D}$ phase correlation method which is 3 times faster than the original. Applied to SLAM this performance increase allows larger volumes to be processed for the same complexity or correspondingly greater accuracy for a given amount of computation time.

\section{Previous Work}




\subsection{Monocular Camera Feature Based Systems}

Monocular Feature based SLAM systems use feature matches to estimate camera pose and location changes across frames [4]. Variations of this method use different features including: corners and lines [14], image patches [25] and exemplar feature matching [3]. SIFT features are used most often in $\operatorname{SLAM}[1,7,13,22]$, in addition FAST features have been explored [1619]. Beall et al [1] made use of both SIFT and SURF features in their underwater SLAM system. Real-time monocular SLAM systems based on this approach have also been proposed $[3,22]$. RANSAC is often used in monocular SLAM $[7,16-18,24]$ to remove outliers which cause incorrect camera parameter estimates. Bundle adjustment is also used as an additional step to refine camera parameter estimation [7].

\subsection{Stereo Camera Feature Based Systems}

Stereo based SLAM systems also use features to estimate camera parameters. However, stereo based systems are capable of generating dense depth data more easily using stereo algorithms. Miro et al [20] proposed a stereo based method which uses SIFT and the extended Kalman filter. The method by Van Gool et al [23] works with un-calibrated stereo pairs. It uses Harris corner features and a multi-view stereo algorithm. Sim et al [26] and Gil et al [9] both presented stereo based SLAM systems which use SIFT.

\subsection{RGB-D Sensor Feature Based Systems}

RGB-D SLAM systems use both depth and image data and are capable of generating dense 3D reconstructions. Many of these methods rely on feature matching techniques $[5,6,11]$. RANSAC is often used to filter outliers for the estimation of camera parameters[5, 6 , 11]. Another method which has also been used extensively in the area is Iterative Closest Point (ICP) $[2,6,11,12,21,27]$. ICP iteratively registers point cloud data, and is used to refine camera parameter estimates. A method named KinectFusion was proposed by Newcombe et al [21] which uses RANSAC and a GPU implementation of IPC. Whelan et al [29] extended this method allowing it to map larger areas using Fast Odometry From Vision (FOVIS) over ICP. Bylow et al [2] improved the ICP approach by registering data using a signed distance function.

\subsection{Non-Feature Based Methods}

Several RGB-D SLAM systems are also non-feature based $[12,15,28]$. Weikersdorfer et al [28] presented a novel sensor system named D-eDVS along with an event based SLAM algorithm. The D-eDVS sensor combines depth and event driven contrast detection. Rather than using features, it uses all detected data for registration.
Kerl et al [15] proposed a dense RGB-D SLAM system which uses a probabilistic camera parameter estimation procedure. It uses the entire image rather than features to perform SLAM.

\subsection{Summary}

As is evident from the current literature, SLAM typically relies on feature matching and RANSAC. However, these approaches fail when there are too few features, when feature confusion occurs or, when features are non-stationary due to object motion. As the extent of random feature displacement becomes more global the effectiveness of these approaches diminishes. Feature matching also dominates in image registration. However, Fourier based methods have been shown to work well under larger rotations and scales [10] whilst being closed form, insensitive to object motion and scaling naturally to GPU implementations. Accordingly, we propose a novel, closed form Fourier based SLAM method.

\section{Method}

The proposed SLAM method consists of various steps. First each frame $f_{i}$ that is captured, consisting of a colour and depth image pair is projected into 3D space, forming colour point cloud points $s_{i}$ and re-sampled into a volume $V_{i}$. Then, the transform parameters between pairs of volumes $V_{i}$ and $V_{i+1}$ are estimated using

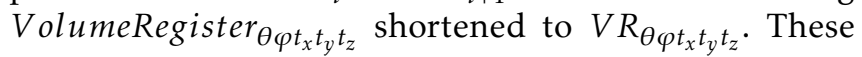
parameters are used to update transformation matrix $M$. The points corresponding to $f_{2}$ (points ${ }_{1}$ ) are then transformed using the updated $M$ matrix and added to the cumulative PointCloud database. Two lists, Cameras and Poses, are also updated to track camera pose and location per frame. This basic procedure is given in listings 1 and elaborated upon in subsequent subsections.

\subsection{Sensor Input}

The input to our method is a color and depth image pair, $f(u, v)$ and $g(u, v)$ obtained using an Asus Xtion PRO LIVE sensor at a resolution of $640 \times 480$. Each pixel is projected into $3 \mathrm{D}$ space using $X_{u, v}=\frac{\left(u-c_{x}\right) Z_{u, v}}{f}, Y_{u, v}=$ $\frac{\left(v-c_{y}\right) Z_{u, v}}{f}$ and $Z_{u, v}=g(u, v)$. Here, $\left[c_{x} c_{y}\right]^{T}$ represent the center of the image whilst $f$ represents the focal length, defined as 525.0. The point clouds generated by projecting these images are then quantized into image volumes. Results reported in this paper were obtained using volumes of $384^{3}$ voxels in size.

\subsection{Volume Registration}

Figure 1 shows a functional block diagram of our method. The input data are two 3D volumes 
Listing 1. Phase Correlation Based SLAM Algorithm

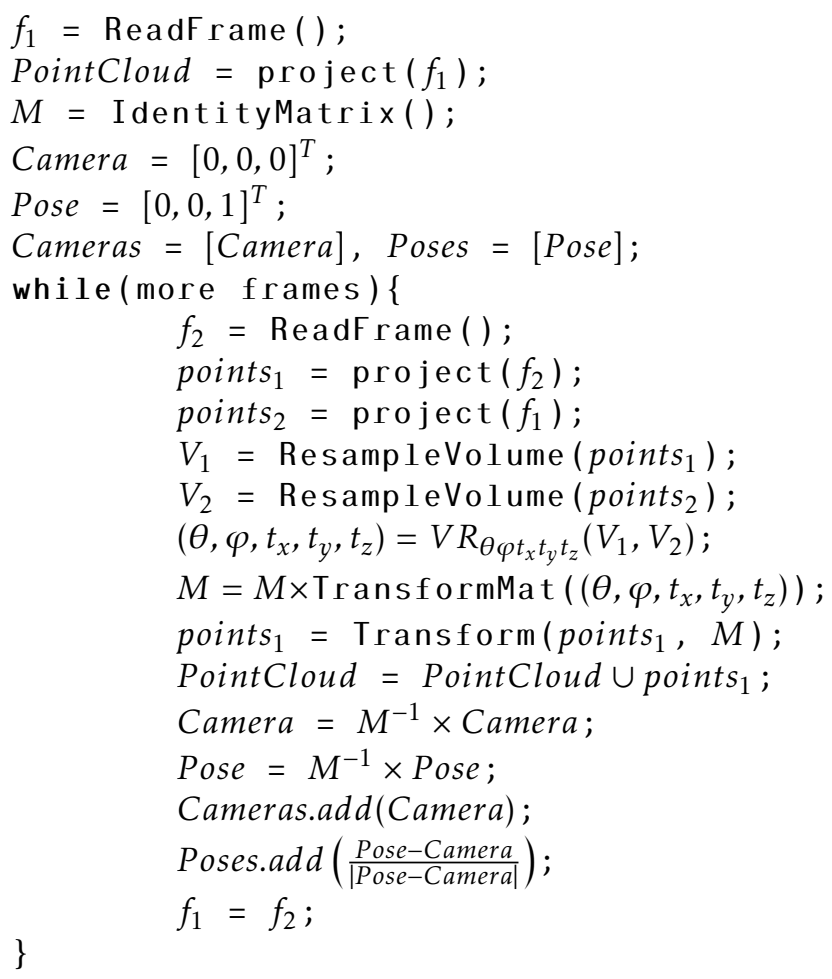

(Volume $_{1}$ and Volume $_{2}$ ) and the output is the transformation matrix required to register the two volumes. The volumes are first Hanning windowed. Next, a translation independent representation is obtained for each by taking the magnitude of their $3 \mathrm{D}$ FFTs. Then a $\log$ function is applied to the resulting magnitude values, improving scale and rotation estimation [10]. Following a log-spherical transformation, 3D phase correlation is performed to find the global rotation and scale relationship between Volume $_{1}$ and Volume $_{2}$. Volume 1 is then inversely transformed by the rotation and scale parameters, leaving only the translation to be resolved. This is found by applying phase correlation again between the transformed Volume V $_{1}$ and Volume V .

\subsection{Phase Correlation}

Given a volume $V_{1}$ and a spatially shifted version of it $V_{2}$, the offset can be recovered using PhaseCorrelation (Eq. 1). This function takes two volumes as input and returns the translation between them.

$$
(x, y, z)=\text { PhaseCorrelation }\left(V_{m}, V_{n}\right)
$$

The PhaseCorrelation function first applies 3D FFTs to volumes, $V_{1}$ and $V_{2}$, converting them into the frequency domain, i.e. $F_{1_{x, y, z}}=\operatorname{FFT}\left(V_{1}\right)$ and $F_{2_{x, y, z}}=$ $\operatorname{FFT}\left(V_{2}\right)$. Taking the normalised cross power spectrum using Eq. 2 completes the Phase correlation function.

$$
F_{3_{x, y, z}}=\frac{F_{1_{x, y, z}} \circ F_{2_{x, y, z}^{*}}^{*}}{\left|F_{1_{x, y, z}} \circ F_{2_{x, y, z}}^{*}\right|}
$$

Here, $\circ$ is an element-wise multiplication and $|x|$ is the magnitude function. Taking the inverse FFT of $F_{3}$, gives the phase correlation volume $V_{3}\left(V_{3}=F F T^{-1}\left(F_{3}\right)\right)$. The location of the peak value in $V_{3},\left(x_{1}, y_{1}, z_{1}\right)$ gives the shift between the $V_{1}$ and $V_{2}$. The phase correlation volume is typically noisy making the peak difficult to locate.

\subsection{Recovering Scale, Rotation and Translation Parameters}

If $V_{1}$ and $V_{2}$ are instead rotated and scaled versions of the same volume, such that they are related by some translation $\left(t_{x}, t_{y}, t_{z}\right)$, y-axis rotation $\theta$, and scale $\varphi$. Further action is required to recover translation parameters. The first step, given two volumes $V_{1}$ and $V_{2}$ of size $N^{3}$ is to apply a Hanning windowing function (Eq. 3).

$$
H W_{x, y, z}=\frac{1}{2}\left(1-\cos \left(\frac{2 \pi\left(\sqrt{\left(\frac{N}{2}\right)^{3}}-\sqrt{\left(x-\frac{N}{2}\right)^{2}+\left(y-\frac{N}{2}\right)^{2}+\left(z-\frac{N}{2}\right)^{2}}\right)}{2 \sqrt{\left(\frac{N}{2}\right)^{3}}-1}\right)\right)
$$

The rotation and scale factors are recovered first using a translation independent representation of the volumes using the Fourier shift theory. For this, the magnitude of the FFT of the volumes is taken, $M_{1}=\left|F F T\left(V_{1}\right)\right|$, $M_{2}=\left|F F T\left(V_{2}\right)\right|$. The zero-frequency of both $M_{1}$ and $M_{2}$ is shifted to the center of the volume and the $\log$ of the result is taken $M_{1}^{\prime}=\log \left(M_{1}\right), M_{2}^{\prime}=\log \left(M_{2}\right)$ which reduces noise on the phase correlation volume. A log-spherical transform is then used to turn rotation and scaling into translation for both $M_{1}^{\prime}$ and $M_{2}^{\prime}$. Eq. 4 shows the corresponding log-spherical space coordinate $\left(X_{\text {log-spherical }}, Y_{\text {log-spherical }}, Z_{\text {log-spherical }}\right)$ for a given $(x, y, z)$ euclidean space coordinate.

$$
\begin{aligned}
X_{\log -\text { spherical }} & =\frac{\operatorname{atan}\left(\left(\frac{x-\frac{N}{2}}{\sqrt{x^{2}+y^{2}+z^{2}}}\right)\left(\frac{y-\frac{N}{2}}{\sqrt{x^{2}+y^{2}+z^{2}}}\right)^{-1}\right) N}{360} \\
Y_{\log \text {-spherical }} & =\frac{\operatorname{acos}\left(\frac{y}{\sqrt{x^{2}+y^{2}+z^{2}}}\right) N}{180} \\
Z_{\log \text {-spherical }} & =\frac{\log \left(\sqrt{x^{2}+y^{2}+z^{2}}\right) N}{\log \left(\frac{N}{2.56}\right)}
\end{aligned}
$$

The log-spherical transforms of $M_{1}^{\prime}$ and $M_{2}^{\prime}$ are then phase correlated to find the shift between them, $\left(x_{M^{\prime}}, y_{M^{\prime}}, z_{M^{\prime}}\right)=$ PhaseCorrelation $\left(M_{1}^{\prime}, M_{2}^{\prime}\right)$. The rotation $\theta$ and scale $\varphi$ factors between $V_{1}$ and $V_{2}$ can 


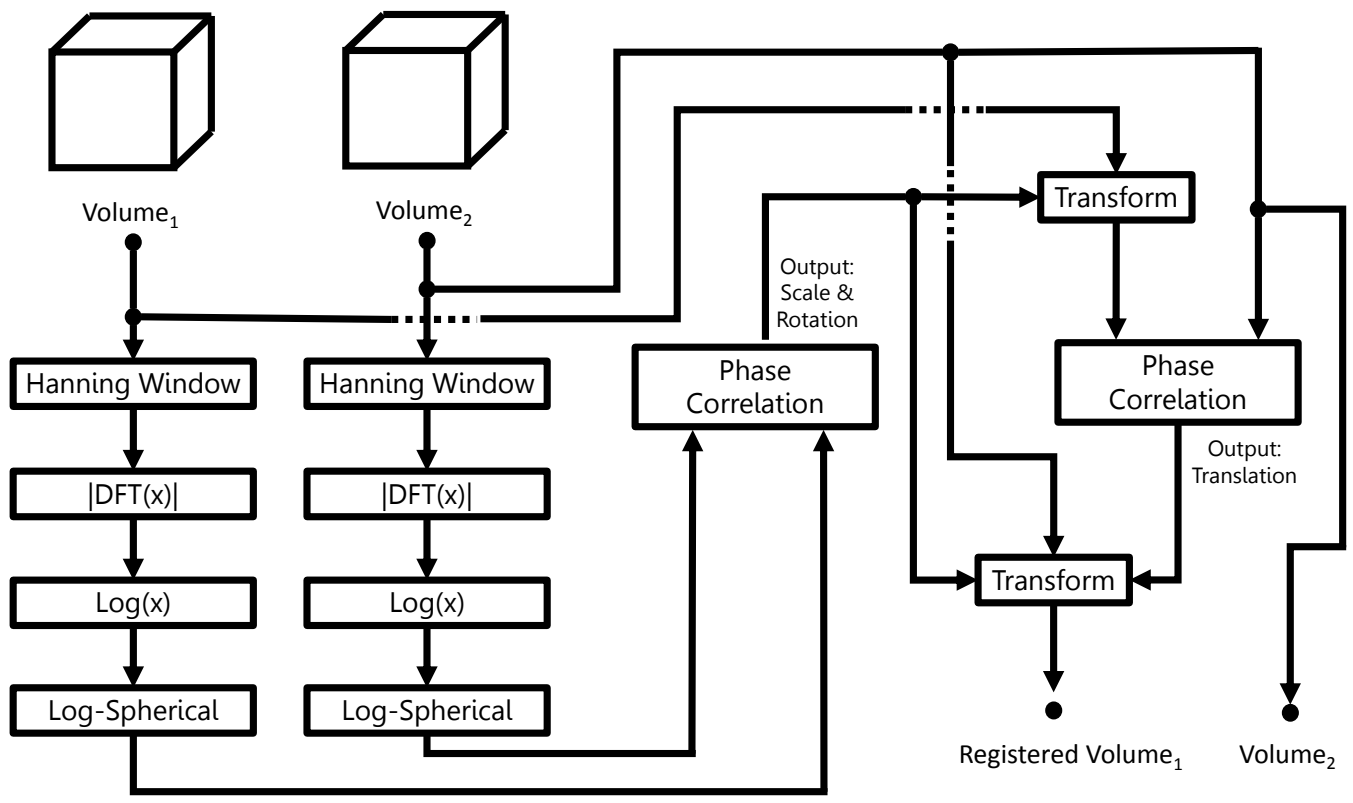

Figure 1. System Diagram for Registration Process

then be found from the shift parameters using Eq. 5 .

$$
\begin{aligned}
\theta & =\frac{-360 x_{M^{\prime}}}{N} \\
\varphi & =e^{-\left(2.56^{-1} N\right) z_{M^{\prime}} N^{-1}}
\end{aligned}
$$

Using $\theta$ and $\varphi, V_{1}$ can now be inverse transformed (using $\left(\frac{N}{2}, \frac{N}{2}, \frac{N}{2}\right)$ as the origin). This aligns $V_{1}$ and $V_{2}$ with respect to scale and $y$-axis rotation. The translation parameters $\left(t_{x}, t_{y}, t_{z}\right)$ can then be found using phase correlation as given in Eq. 6.

$\left(t_{x}, t_{y}, t_{z}\right)=$ PhaseCorrelation $\left(\operatorname{scale}\left(\right.\right.$ rotate $\left.\left.\left(V_{1}, \theta\right), \varphi\right), V_{2}\right)$

The complete function to recover translation, rotation and scaling, combining equations $2-6$ as is denoted in 1 is 7 .

$$
\left(\theta, \varphi, t_{x}, t_{y}, t_{z}\right)=\text { PhaseCorrelation } \theta \varphi t_{x} t_{y} t_{z}\left(V_{m}, V_{n}\right)
$$

\section{Improving Performance}

To reduce complexity we focus on areas which require the most computation time. In the earlier defined Fourier based reconstruction technique, this occurs in the two 3D phase correlations which need to be computed. We describe the method of reduction here; a block diagram for this technique is given in figure 2. We refer to this method as fast volume registration (FVR) in reference general volume registration (VR). The speedup begins by computing the 3D DFT of both input volumes and taking the magnitude of these. Rather than directly performing a 3D log-spherical transform and a 3D phase correlation operation on these volumes, we use a novel transform we call a spherical-map transform (details in 4.1).

This transform converts rotation into translation whilst simultaneously unfolding the 3D space down to 2-dimensions. After this, a 2D phase correlation that requires significantly less processing compared with the 3D counterpart is used to compute the rotation. Next, having obtained the rotation parameter, the rotation is eliminated from the transformation by rotating the first volume by this parameter. The two volumes are then passed through two orthogonal projection mapping functions. This also converts the volumes to 2D space. We use two transforms for both volumes, one projection along the $\mathrm{x}$-axis, another along the $\mathrm{z}$-axis. Once the $\mathrm{x}$-axis projections of both volumes are complete, we can do another 2D phase correlation to give us the $\mathrm{z}$-translation. The $2 \mathrm{D}$ phase correlation of the $\mathrm{z}$-axis projections gives us the $\mathrm{x}$ and $\mathrm{y}$ axis translations separating the original volumes. The final output of this method gives the rotation and translational shifts between the input volumes. The projections add little complexity to the overall algorithm and since 2D phase correlation operations are used in place of $3 \mathrm{D}$ ones, much computation time is reduced.

\subsection{Spherical-map transform}

The spherical map transform both reduces the $3 \mathrm{D}$ volume to a $2 \mathrm{D}$ image, and any rotation about the $\mathrm{y}$-axis becomes $\mathrm{x}$-axis translation in the output image. 


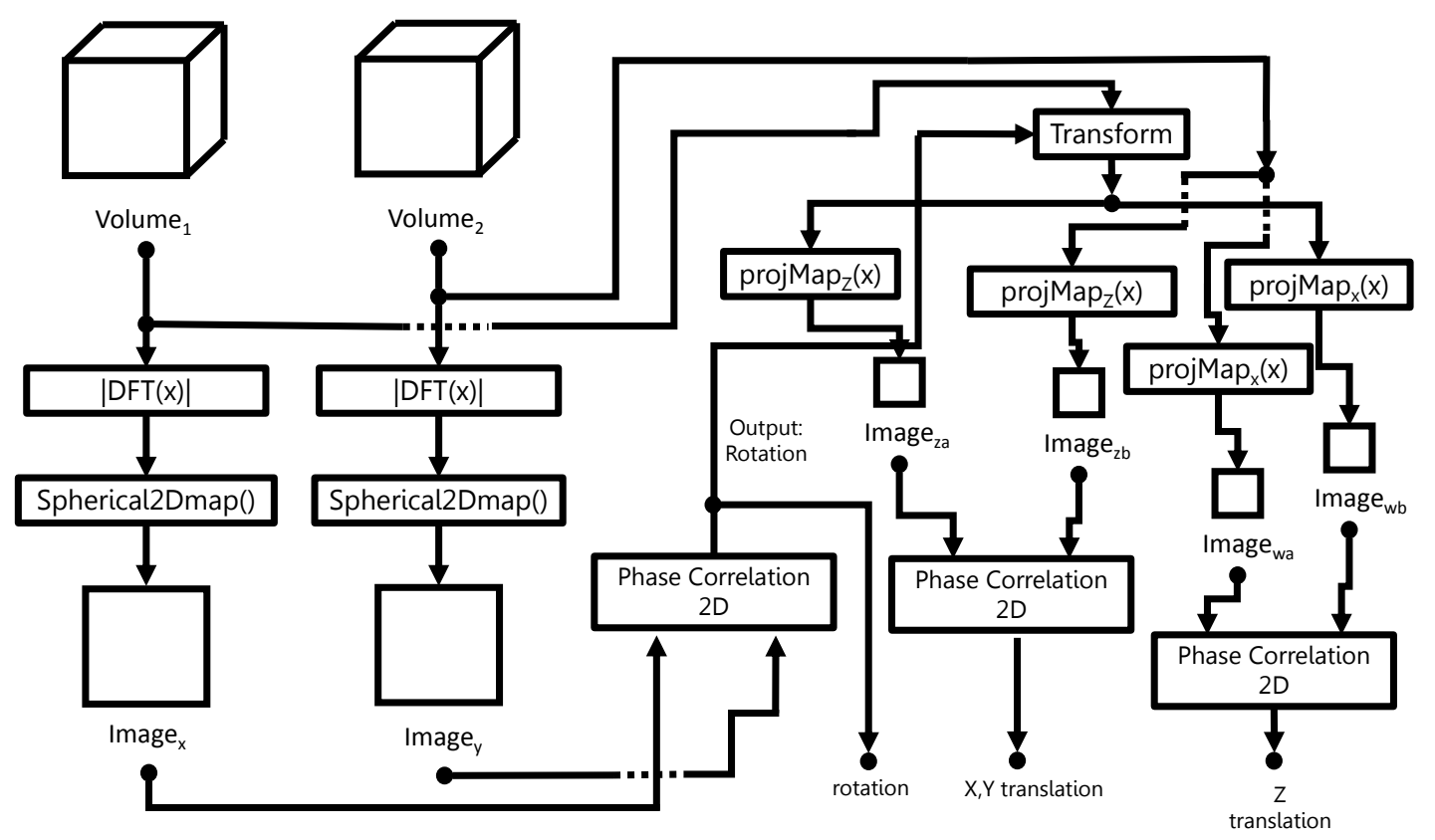

Figure 2. System Diagram for Fast Volume Registration

An example of the bunny model and the sphericalmap transform of this model is given in figure 3 , the mathematics are defined in equations 8 and 9. Given a coordinate in 2D Cartesian space $x, y$, we compute the ray $\left[R a y_{x} R_{a y} R_{a y}\right]^{T}$ from the volume center and sum up the voxel values along the ray (equation 9).

$$
\begin{gathered}
\operatorname{Ray}_{x}(x, y)=\cos \left(\frac{360 x}{N}\right) \sin \left(\frac{180 y}{N}\right)+\frac{N}{2} \\
\operatorname{Ray}_{y}(y)=\cos \left(\frac{180 y}{N}\right)+\frac{N}{2} \\
\operatorname{Ray}_{z}(x, y)=\sin \left(\frac{360 x}{N}\right) \sin \left(\frac{180 y}{N}\right)+\frac{N}{2} \\
\operatorname{Im}_{x, y}=\sum_{r=1}^{\left(2^{-1} N\right)^{1.5}} \operatorname{Vol}\left(\operatorname{Ray}_{x}(x, y) r, \operatorname{Ray}_{y}(y) r, \operatorname{Ray}_{z}(x, y) r\right)
\end{gathered}
$$

This is process essentially sums up the values along a given ray defined by scaling spherical coordinates and adding up the values intersecting the ray. The resulting image, maps $3 \mathrm{D}$ y-axis rotation to $2 \mathrm{D} \mathrm{x}$-axis translation.

\subsection{Projection-map transform}

The projection map transform is similar to an orthogonal projection of the volume along some given axis. For the projection map transform, given an output image $I m_{a}$ and an input volume $V o l_{a}$, each pixel in $I m_{a}$ is defined mathematically as the summation of values along a particular axis given the image coordinates. The $\mathrm{x}$-axis transform and the $\mathrm{Z}$-axis transform are defined in equations 10 and 11 respectively.

$$
\begin{aligned}
& \operatorname{Im}(z, y)=\sum_{x=0}^{N} V o l_{a}(x, y, z) \\
& \operatorname{Im}(x, y)=\sum_{z=0}^{N} V o l_{a}(x, y, z)
\end{aligned}
$$

The process defined by equation 10 maps 3D z-axis translation to $2 \mathrm{D} \mathrm{x}$-axis translation, whilst equation 11 maps $3 \mathrm{D} x$-axis and $y$-axis translation into $2 \mathrm{D} x$-axis and $\mathrm{y}$-axis translation.

\section{Performance Analysis}

\subsection{D Phase Correlation Performance}

To assess the performance of our method, the size of the volumes being registered is defined as $N^{3}$ whilst each frame is sampled at a resolution of $W$ $\times H$. The projection process requires $12 \mathrm{WH}$ operations whilst re-sampling the point cloud requires $2 W H$ operations. The Volume Registration process, VolumeRegister $\theta \varphi t_{x} t_{y} t_{z}\left(V_{1}, V_{2}\right)$ consists of $2 \times$ Hanning windowing processes, $2 \times 3 \mathrm{D}$ FFTs, $2 \times$ volumelogs, $2 \times \log$-spherical transforms, $2 \times$ phase correlation processes and $1 \times$ linear transformation and peak finding. 


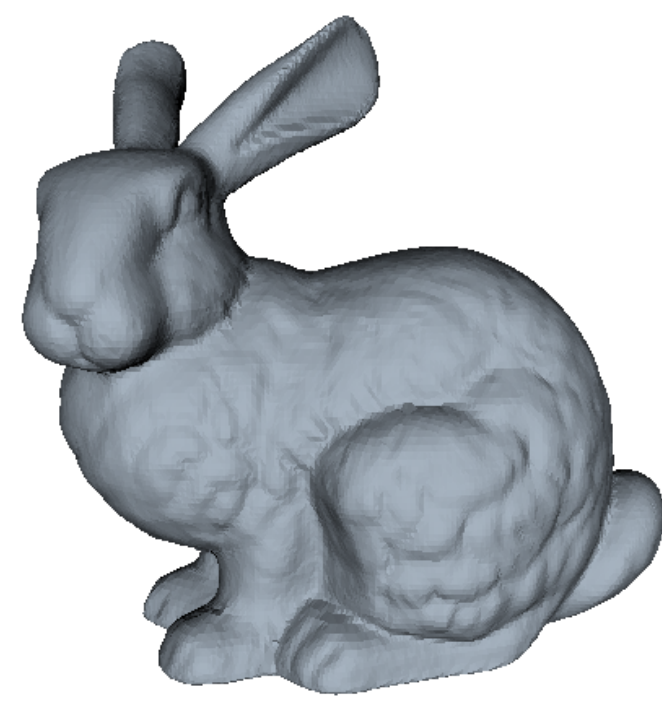

(a) original

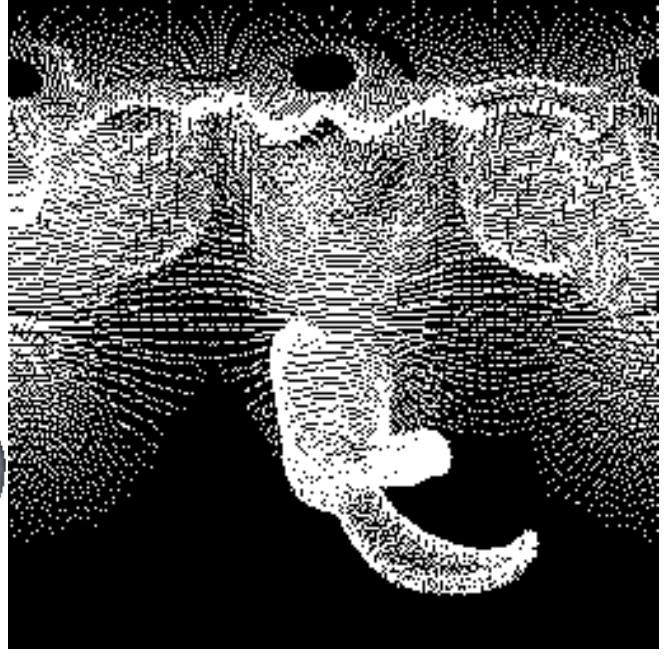

(b) transform

Figure 3. The Spherical Map Transform.

The Hanning windowing function requires 26 operations. The 3D FFT has complexity of $3 N^{3} \log N$, the $\log$ and $\log$-spherical transform functions require 3 and 58 operations per voxel respectively. Multiplying two frequency spectra together and transforming a volume requires 15 and 30 operations per voxel respectively. Finding the peak value requires $2 \mathrm{~N}^{3}$ operations. The complexity in terms of number of operations for the phase correlation process is given in Eq. 12 This process requires $2 \times 3$ D FFTs, $1 \times$ frequency spectra multiplication, and $1 \times$ peak finding operation.

$$
6 N^{3} \log N+2 N^{3}+15
$$

The total complexity can then be found by taking into account the projection and re-sampling totals as well as the total for VolumeRegister $\theta \varphi t_{x} t_{y} t_{z}\left(V_{1}, V_{2}\right)$. Tallying the number of operations for each process and multiplying them by number of times the process is performed gives us the number of operations as a function of $W, H$ and $N$ in Eq. 13.

$$
6 N^{3}+28 W H+18\left(N^{3} \log N\right)+230
$$

\subsection{Analysis of Speed Improvement}

To compare performance of the generic volume registration method with the speed up, we use the complexity defined in equation 13. Here, we ignore the cost of projecting the depth map. The 3D DFT has complexity $3 N^{3} \log (N)$. This is the first step (see figure 2), the next is the spherical-map transform which is complexity $45 N^{3}$. If processed on the GPU the performance becomes 45 operations per voxel assuming that one voxel is assigned to one unit of processing. A 3D transform is 30 operations per voxel, 2D phase correlation requires 15 operations to multiply the frequency spectra and $2 N^{2} \log (N)$ operations to do the 2D FFT. Finally a projection map transform requires 1 operation per voxel.

In total, the proposed method requires $2 \times 3 \mathrm{D}$ FFTs, $2 \times$ spherical-map transforms, $1 \times 3 \mathrm{D}$ geometrical transformation, $3 \times 2 \mathrm{D}$ phase correlations and $4 \times$ projection map transforms. The total complexity is added up for all of these functions and given in equation 14 .

$$
6 \log (N) \times\left(N^{3}+N^{2}\right)+169
$$

Figure 5 provides a visualization of the performance improvement which the proposed method achieves over the original Fourier volume registration approach. It is clear that the proposed method is around 3 times faster than the original Fourier based volume registration approach. This is due to the reduction in the amount of data to process afforded by the novel spherical-map transform and orthogonal projection methods.

\section{Experiments}

[ht]

A number of experiments were undertaken to assess the reconstruction accuracy, noise sensitivity and robustness to object motion. Experiments were performed using an ASUS Zenbook UX303LN with an Intel i7 $5500 \mathrm{u}$ Dual Core $2.4 \mathrm{GHz}$ processor, 8GB of RAM and an NVIDIA GE-FORCE 840 M GPU. 


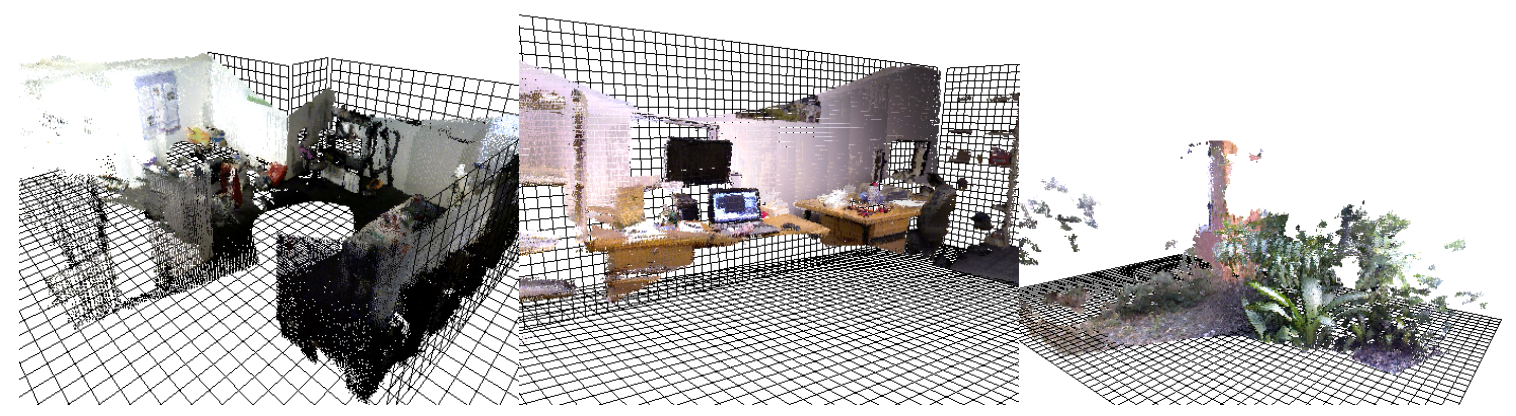

(a) Apartment

(b) Office

(c) Garden

Figure 4. Reconstructed Scenes.

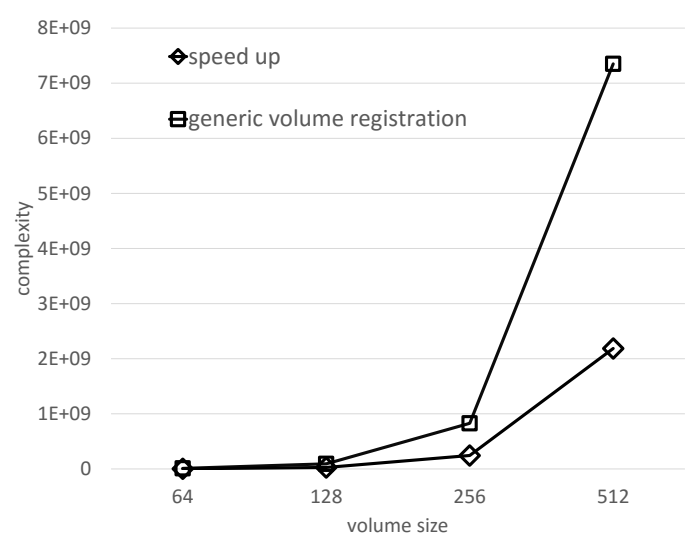

Figure 5. Comparison of performance between volume registration and the proposed speed up for different volume sizes.

\begin{tabular}{ccccc}
\hline translation $(\mathbf{c m})$ & noise range $\mathbf{( \% )}$ & SNR & error $(\mathbf{c m})$ & error (voxel) \\
\hline $5 \mathrm{~cm}$ & 0 & $\infty$ & 0 & 0 \\
$5 \mathrm{~cm}$ & 10 & $20 \mathrm{db}$ & 0 & 0 \\
$5 \mathrm{~cm}$ & 25 & $12 \mathrm{db}$ & 0 & 0 \\
$5 \mathrm{~cm}$ & 50 & $6 \mathrm{db}$ & 0 & 0 \\
$5 \mathrm{~cm}$ & 75 & $2.5 \mathrm{db}$ & 112.28 & 89.83 \\
$10 \mathrm{~cm}$ & 0 & $\infty$ & 0 & 0 \\
$10 \mathrm{~cm}$ & 10 & $20 \mathrm{db}$ & 0 & 0 \\
$10 \mathrm{~cm}$ & 25 & $12 \mathrm{db}$ & 0 & 0 \\
$10 \mathrm{~cm}$ & 50 & $6 \mathrm{db}$ & 156.65 & 125.32 \\
$15 \mathrm{~cm}$ & 0 & $\infty$ & 2.8 & 2.24 \\
$15 \mathrm{~cm}$ & 10 & $20 \mathrm{db}$ & 2.8 & 2.24 \\
$15 \mathrm{~cm}$ & 25 & $12 \mathrm{db}$ & 2.8 & 2.24 \\
$15 \mathrm{~cm}$ & 50 & $6 \mathrm{db}$ & 198.55 & 158.84
\end{tabular}

Table 1. Translation Tracking

For volumes of $384^{3}, 1 \times$ registration per second was possible. To achieve real-time performance, 1 out of every 30th frames was processed.

[ht]

\subsection{Reconstruction Quality}

To assess reconstruction accuracy, two indoor environments (Apartment and Office) as well as one outdoor environment (Garden) were used, these can be seen in figures $4 \mathrm{a}, 4 \mathrm{~b}$ and $4 \mathrm{c}$ respectively. The Apartment reconstruction was recorded by moving through a room whilst rotating the camera. Some frames contained nothing but featureless walls, others had contrast shifts due to the camera's automatic contrast feature, yet, accurate reconstruction was achieved. The office reconstruction was generated by rotating the camera about the y-axis while moving backwards. Whilst our method is a closed form solution, its accuracy is still comparable to existing feature based SLAM methods. Typical feature based methods work well with indoor environments where local features are readily distinguishable and easy to match. They do not tend to work as well with complex outdoor scenes where feature confusion is likely. To assess performance in such outdoor scenes, a garden scene containing bushes, plants and a ground covering of bark and rocks was used. In the case of a feature matching approach this scene would likely result in feature confusion, making camera tracking difficult. The proposed method was able to produce a good quality reconstruction. Hence, our approach readily overcomes difficulties common to feature matching methods.

\subsection{Noise Sensitivity}

To assess robustness to noise, the estimated camera parameters are compared to ground truth data under different noise conditions. In each experiment, varying amounts of random noise were added per voxel prior to registration. This is expressed in decibels using the Signal to Noise Ratio (SNR). Each voxel value lies in the range [0-1]. Here, a noise value of $10 \%$ means random noise was added in the range $[-0.05$, 0.05]. Tracking error is measured in centimetres and voxel error (the error in the phase correlation volume). The first experiment evaluated noise robustness whilst the camera was translated by varying amounts $(5 \mathrm{~cm}$, $10 \mathrm{~cm}$ and $15 \mathrm{~cm})$. Results in Table 1 show that, for camera translations up to $15 \mathrm{~cm}$ and SNR values above 6.0 our method is robust to noise. At video rates, a 


\begin{tabular}{ccccc}
\hline rotation & noise $(\%)$ & SNR & error $(\theta)$ & error (voxel) \\
\hline $10^{\circ}$ & 0 & $\infty$ & 0.31 & 0 \\
$10^{\circ}$ & 10 & $20 \mathrm{db}$ & 0.31 & 0 \\
$10^{\circ}$ & 25 & $12 \mathrm{db}$ & 0.63 & 1 \\
$10^{\circ}$ & 30 & $10.5 \mathrm{db}$ & 90.62 & 96 \\
$20^{\circ}$ & 0 & $\infty$ & 0.31 & 0 \\
$20^{\circ}$ & 10 & $20 \mathrm{db}$ & 0.63 & 1 \\
$20^{\circ}$ & 15 & $16.5 \mathrm{db}$ & 38.13 & 40 \\
$30^{\circ}$ & 0 & $\infty$ & 3.75 & 4 \\
$30^{\circ}$ & 10 & $20 \mathrm{db}$ & 3.28 & 3 \\
$30^{\circ}$ & 15 & $16.5 \mathrm{db}$ & 30 & 32
\end{tabular}

Table 2. Rotation Tracking

\begin{tabular}{ccc}
\hline Object Size & error $(\mathbf{c m})$ & error (voxel) \\
\hline 0.35 & 0 & 0 \\
2.95 & 0 & 0 \\
6.22 & 0 & 0 \\
12.28 & 0 & 0 \\
19.82 & 0 & 0 \\
22.39 & 0 & 0 \\
26.09 & 0 & 0 \\
31.00 & 0 & 0 \\
48.23 & 38.42 & 15 \\
74.32 & 113.57 & 44
\end{tabular}

Table 3. Object Motion Test displacement of $10 \mathrm{~cm}$ per frame equates to a camera velocity of $3 \mathrm{~m} / \mathrm{s}$ (about twice the normal walking speed).

Table 2 shows the results for tracking camera rotations of 10, 20 and 30 degrees per frame. At video rates, 12 degrees per frame is almost a full rotation per second. In rotations of 10 degrees, the error was less than a degree for all but a noise level of $30 \%$ and above. This base line error is due to the sampling resolution of the volume, as voxel error was in fact zero. As with pure translation, the effect of noise increases with camera disparity. At 30 degrees, little matching information is available. However, for noise levels of $10 \%$ or less, voxel distance error was as low as 4 with an angular error less than 3.8. Rotations of this magnitude are unlikely, moreover motion blur would occur.

\subsection{Robustness to Object Motion}

To assess robustness to object motion, experiments were conducted by moving the camera backwards along the $\mathrm{z}$-axis by $5 \mathrm{~cm}$ per frame whilst moving objects in and out of the scene so that they only appear in one of the volumes being registered. Various sized objects including stacks of CDs, large boxes, people and several pieces of furniture were used and are measured by the percentage of the frame they occupy. Results from Table 3 show the proposed method was accurate upto an object size of $31 \%$, but failed for objects taking up over $48.23 \%$.

\section{Conclusions}

In this paper, we proposed a novel non-feature based approach to SLAM, which can generate accurate 3D color reconstructions of both indoor and outdoor environments. This method is a closed form solution, scales naturally to the GPU, and is shown to be robust to global noise and object motion. We also proposed a method to speed up this method by up to 3 times.

\section{Future Work}

Future work in this area includes investigating a system to recover from mis-registered frames and to continue to improve performance.

Acknowledgement. Luke Lincoln would like to thank Thao Lincoln.

\section{References}

[1] Beall, C., Dellaert, F., Mahon, I. and Williams, S.B. (2011) Bundle adjustment in large-scale 3d reconstructions based on underwater robotic surveys. In OCEANS, 2011 IEEE-Spain (IEEE): 1-6.

[2] Bylow, E., Sturm, J., Kerl, C., Kahl, F. and Cremers, D. (2013) Real-time camera tracking and 3d reconstruction using signed distance functions. In Robotics: Science and Systems (RSS) Conference 2013, 9.

[3] Chekhlov, D., Pupilli, M., Mayol, W. and Calway, A. (2007) Robust real-time visual slam using scale prediction and exemplar based feature description. In Computer Vision and Pattern Recognition, 2007. CVPR'07. IEEE Conference on (IEEE): 1-7.

[4] Davison, A.J. and Murray, D.W. (2002) Simultaneous localization and map-building using active vision. Pattern Analysis and Machine Intelligence, IEEE Transactions on 24(7): 865-880.

[5] Endres, F., Hess, J., Engelhard, N., Sturm, J., Cremers, D. and Burgard, W. (2012) An evaluation of the rgb-d slam system. In Robotics and Automation (ICRA), 2012 IEEE International Conference on (IEEE): 1691-1696.

[6] Engelhard, N., Endres, F., Hess, J., Sturm, J. and Burgard, W. (2011) Real-time 3d visual slam with a hand-held rgb-d camera. In Proc. of the RGB-D Workshop on $3 D$ Perception in Robotics at the European Robotics Forum, Vasteras, Sweden, $\mathbf{1 8 0 .}$.

[7] Eudes, A., Lhuillier, M., Naudet-Collette, S. and Dноме, M. (2010) Fast odometry integration in local bundle adjustment-based visual slam. In Pattern Recognition (ICPR), 2010 20th International Conference on (IEEE): 290-293.

[8] Foroosh, H., Zerubia, J.B. and Berthod, M. (2002) Extension of phase correlation to subpixel registration. Image Processing, IEEE Transactions on 11: 188-200.

[9] Gil, A., Reinoso, O., Mozos, O.M., Stachniss, C. and Burgard, W. (2006) Improving data association in 
vision-based slam. In Intelligent Robots and Systems, 2006 IEEE/RSJ International Conference on (IEEE): 2076-2081.

[10] Gonzalez, R. (2011) Improving phase correlation for image registration. In Image And Vision Computing New Zealand 2011 (IVCNZ2011) (http://ieeexplore. ieee. org/xpl/conhome. jsp? punumber $=1002602$ ).

[11] Henry, P., Krainin, M., Herbst, E., Ren, X. and Fox, D. (2010) Rgb-d mapping: Using depth cameras for dense $3 \mathrm{~d}$ modeling of indoor environments. In In the 12th International Symposium on Experimental Robotics (ISER (Citeseer).

[12] Izadi, S., Kim, D., Hilliges, O., Molyneaux, D., Newcombe, R., Kohl, P., Shotton, J. et al. (2011) Kinectfusion: real-time $3 \mathrm{~d}$ reconstruction and interaction using a moving depth camera. In Proceedings of the 24th annual ACM symposium on User interface software and technology (ACM): 559-568.

[13] Jensfelt, P., Kragic, D., Folkesson, J. and Bjorkman, M. (2006) A framework for vision based bearing only 3d slam. In Robotics and Automation, 2006. ICRA 2006. Proceedings 2006 IEEE International Conference on (IEEE): 1944-1950.

[14] Jeong, W.Y. and Lee, K.M. (2006) Visual slam with line and corner features. In Intelligent Robots and Systems, 2006 IEEE/RSJ International Conference on (IEEE): 25702575.

[15] Kerl, C., Sturm, J. and Cremers, D. (2013) Dense visual slam for rgb-d cameras. In Intelligent Robots and Systems (IROS), 2013 IEEE/RSJ International Conference on (IEEE): 2100-2106.

[16] Konolige, K. and Agrawal, M. (2008) Frameslam: From bundle adjustment to real-time visual mapping. Robotics, IEEE Transactions on 24(5): 1066-1077.

[17] Konolige, K., Bowman, J., Chen, J., Mihelich, P., Calonder, M., Lepetit, V. and Fua, P. (2010) View-based maps. The International Journal of Robotics Research .

[18] Kundu, A., Krishna, K.M. and Jawahar, C. (2010) Realtime motion segmentation based multibody visual slam. In Proceedings of the Seventh Indian Conference on Computer Vision, Graphics and Image Processing (ACM): 251-258.

[19] Leelasawassuk, T. and Mayol-Cuevas, W.W. (2013) 3d from looking: using wearable gaze tracking for handsfree and feedback-free object modelling. In Proceedings of the 17th annual international symposium on International symposium on wearable computers (ACM): 105-112.

[20] Miro, J.V., Zhou, W. and Dissanayake, G. (2006) Towards vision based navigation in large indoor environments. In Intelligent Robots and Systems, 2006 IEEE/RSJ International Conference on (IEEE): 2096-2102.

[21] Newcombe, R.A., Izadi, S., Hilliges, O., Molyneaux, D., Kim, D., Davison, A.J., KoHI, P. et al. (2011) Kinectfusion: Real-time dense surface mapping and tracking. In Mixed and augmented reality (ISMAR), 2011 10th IEEE international symposium on (IEEE): 127-136.

[22] Pollefeys, M., Nistér, D., Frahm, J.M., Akbarzadeh, A., Mordohai, P., Clipp, B., Engels, C. et al. (2008) Detailed real-time urban $3 \mathrm{~d}$ reconstruction from video. International Journal of Computer Vision 78(2-3): 143167.

[23] Pollefeys, M., Van Gool, L., Vergauwen, M., Verbiest, F., Cornelis, K., Tops, J. and КосH, R. (2004) Visual modeling with a hand-held camera. International Journal of Computer Vision 59(3): 207-232.

[24] Pradeep, V., Rhemann, C., Izadi, S., Zach, C., Bleyer, M. and Bathiche, S. (2013) Monofusion: Real-time 3d reconstruction of small scenes with a single web camera. In Mixed and Augmented Reality (ISMAR), 2013 IEEE International Symposium on (IEEE): 83-88.

[25] Silveira, G., Malis, E. and Rives, P. (2008) An efficient direct approach to visual slam. Robotics, IEEE Transactions on 24(5): 969-979.

[26] Sim, R., Elinas, P., Griffin, M., Little, J.J. et al. (2005) Vision-based slam using the rao-blackwellised particle filter. In IJCAI Workshop on Reasoning with Uncertainty in Robotics, 14: 9-16.

[27] Stückler, J. and Behnke, S. (2012) Robust real-time registration of rgb-d images using multi-resolution surfel representations. In Robotics; Proceedings of ROBOTIK 2012; 7th German Conference on (VDE): 1-4.

[28] Weikersdorfer, D., Adrian, D.B., Cremers, D. and Conradt, J. (2014) Event-based 3d slam with a depthaugmented dynamic vision sensor. In Robotics and Automation (ICRA), 2014 IEEE International Conference on (IEEE): 359-364.

[29] Whelan, T., Kaess, M., Fallon, M., Johannsson, H., Leonard, J. and McDonald, J. (2012) Kintinuous: Spatially extended kinectfusion. MIT-CSAIL . 